\title{
Two, four, five-shell clusters and colloids
}

\author{
Gïnter Schmid, Norbert Klein, Bernd Morun, Andreas Lehnert \\ Institut für Anorganische Chemie, Universität Essen \\ D-4300 Essen 1, West-Germany \\ Jan-01le Malm, \\ National Center for High Resolution Electron Microscopy, Inorganic Chemistry 2, \\ Chemical Center, Lund, Sweden
}

\begin{abstract}
So-called full-she11 clusters consist of a magic number of metal atoms. Each shell in a cubic (ccp) or hexagonal (hcp) close packed structure contains $10 n^{2}+2$ atoms ( $n=$ number of the shell). The synthesis of two (55 atoms), four ( 309 atoms), and five-shell clusters ( 561 atoms) succeeds by the reduction of appropriate metal complexes with $\mathrm{B}_{2} \mathrm{H}_{6}$ or $\mathrm{H}_{2}$. The clusters can be imaged by high resolution transmission electron microscopy. The chemical and physical properties are remarkably different from those of smaller clusters and of complexes. In continuation of this development gold colloids, considered as very large clusters, can also be stabilized by simple complex ligands and are isolated in solid state. Aqueous collodial solutions are now available in any concentration.
\end{abstract}

\section{INTRODUCTION}

In many cases, the chemistry of an element can be best studied by its elemental structure. Arenes are to be described as derivatives of graphite, six-membered carbon rings and cages with saturated carbon atoms derive from diamond. The higher boranes and carboranes consist of icosahedral fragments or complete icosahedra, the building blocks of the different modifications of elemental boron. Similar examples could be given e.g. for phosphorus, silicon, etc. Transition metal clusters, beginning with 3 , up to hundrets of atoms in most cases can be considered as cutouts of metal lattices. From that point of view, there should be no limitation wether in cluster-size nor in the nature of the metal. From experience we know that noble metals are more suited for cluster formation than others, but numerous examples have also been described with more reactive metals like iron, cobalt, or nickel. Actually, there exists a continous series of clusters up to $M_{13}$ units, the first magic number (ref. 1). Magic numbers are realized, if in cubic ( $c c p$ ) or hexagonal (hcp) close-packed structures completed outer geometries (cubo octahedra or twistet cubo octahedra) are achieved. These particles are also called full-shell clusters, as they can be considered to be constructed by shells, each having $10 n^{2}+2$ atoms $\left(n=1, M_{13}\right)$.

Clusters with more than 13 atoms are also existent but their number is much less numerous (ref. 1). With increasing numbers of atoms the tendency to form ccp or hcp structures also increases. Exceptions are known, e.g. the polyicosahedral structures of mixed gold-silver clusters (ref. 2). Due to their complete outer geometry, full-shell clusters should have a higher stability compared with others. That's why they seem to be formed preferably if metal atoms aggregate as little unhindered as can be. These conditions may be realized in metal vapour or in solution.

\section{RESULTS}

Ful1-shell clusters with the magic number $55(n=2), 309(n=4)$, and $561(n=5)$ can be synthesized by the reduction of different metal complexes by $\mathrm{B}_{2} \mathrm{H}_{6}$ or $\mathrm{H}_{2}$ in solution.

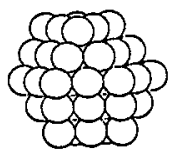

$\mathrm{M}_{5} 5$

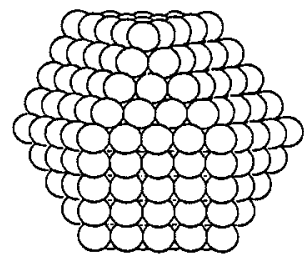

$M_{309}$

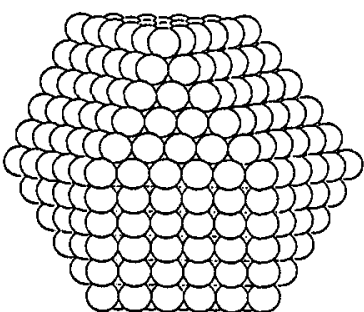

$M_{561}$ 
The generated metal atoms may aggregate to ccp or hcp constructed particles which then must be stabilized by appropriate ligands. The nature as well as the concentration of the ligands is of decisive importance to yield full-shell clusters. It is still not possible to project certain cluster sizes, rather success and failure seem accidental.

Diborane reduction of phosphane and arsane complexes of $\mathrm{Co}, \mathrm{Rh}, \mathrm{Ru}, \mathrm{Pt}$, and $\mathrm{Au}$ in some cases results in $\mathrm{M}_{55} \mathrm{~L}_{12} \mathrm{Cl}_{\mathrm{X}}$ clusters (ref. 3-5). $\mathrm{B}_{2} \mathrm{H}_{6}$ not only serves as reducing agent but also as Lewis acid to bind excessive $\mathrm{PR}_{3}$ and $A s R_{3}$ ligands as $H_{3} B-\mathrm{PR}_{3}$ and $\mathrm{H}_{3} \mathrm{~B}-\mathrm{AsR}_{3}$ adducts. The number of Cl-ligands varies, depending on free coordination positions on the cluster surface. Of more immediate interest are four, and five shell clusters of platinum and palladium (ref. 6-8). If an acetic acid solution of Pt(II)acetate and Phen*

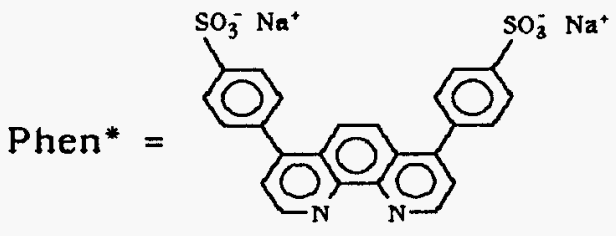

in the molar ratio $7-8: 1$ is at first reacted with hydrogen and after that with oxygen, the four-shell cluster $\mathrm{Pt}_{309} \mathrm{Phen}^{*}{ }_{36} \mathrm{O}_{30} \pm_{10}$ is formed in ca. $30 \%$ yield. It is isolated by centrifugation from aqueous solution (ref.6). By a similar method the five-shell cluster $\mathrm{Pd}_{561}-$ Phen ${ }_{36} \mathrm{O}_{\mathrm{n}}$ (Phen $=$ phenanthroline, $\mathrm{n} \approx 200$ ) can be synthesized (ref. 7), closely related to a Pd five-shell cluster described by Moiseev et al (ref. 8).

The lack of crystals of these large clusters prevents X-ray structure investigations. On the other hand modern high resolution transmission electron microscopy (HRTEM) allows relatively precise size and structure determinations, as is shown representatively in the case of the $\mathrm{Pt}_{30 \mathrm{~g}}$ cluster. Of course, the very exact number of atoms cannot be shown by that method, but the ccp structure as well as the number of atom columns can be observed precisely.

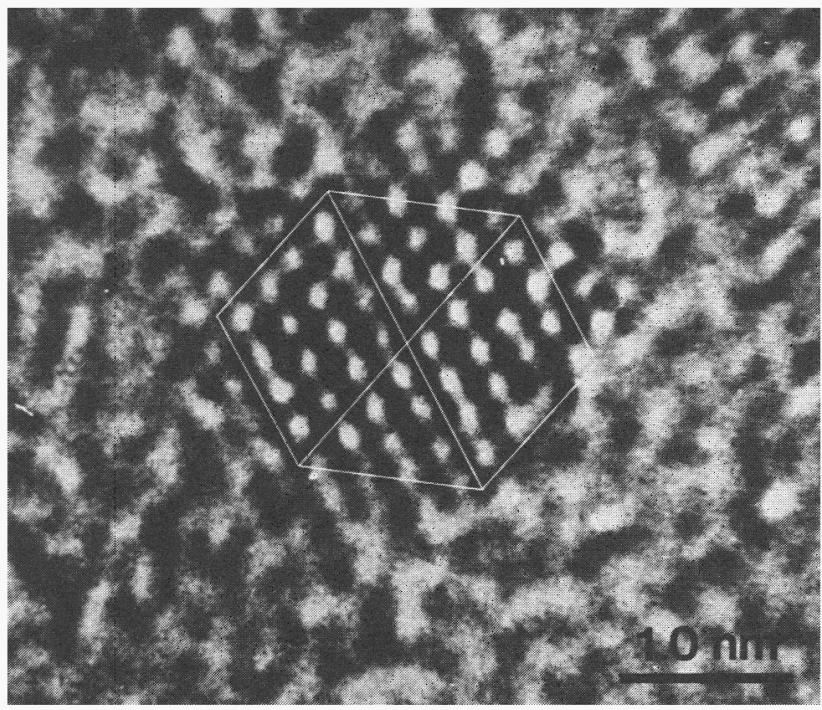

Fig. 1. High resolution transmission electron microscopic image of the $\mathrm{Pt}_{309}$ cluster in the $[110]$ direction.

The size of the cluster nuclei can also be confirmed by X-ray powder diffraction methods. The reflections agree with those of the bulk metals, i.g. cluster and metallic state have the same atomic structure. The half-width of the strongest reflections allows the determination of the particle size. So, in agreement with the calculated values the microscopic images as well as the X-ray determinations result in a diameter of $1,8 \mathrm{~nm}$ for $\mathrm{Pt}_{309}$ and $24-25 \mathrm{~nm}$ for $\mathrm{Pd}_{56_{2}}$. Besides these results, the molecular weights of the complete molecules can be determined with an accuracy of $\pm 10 \%$ by means of an analytical ultracentrifuge.

The expectation of a some time beginning metallic state going from metal to big clusters, is one of the most exciting reasons to be engaged in that field of chemistry and physics. The physical data we so far know do not indicate metallic behaviour, but they show something between molecular and bulk properties leading to the concept of a meta-metal (ref. 9). For lack of clusters larger than 561 atoms, we decided to use the well known gold colloids as very big cluster nuclei and reacted them with complex ligands like the water soluble triphenylphosphane derivative $\mathrm{Ph}_{2} \mathrm{PC}_{6} \mathrm{H}_{4} \mathrm{SO}_{3} \mathrm{Na}$. Gold colloids, prepared according to the well known sodium citrate method (ref. 10) indeed add the phosphane molecules to yield a relatively stable ligand shell which prevents aggregation. By that means the colloids can be iso- 
lated in solid state as golden leafs. They are water soluble in any concentration forming blood red solutions. The used citrate method is described to yield colloids of $20 \mathrm{~nm} \pm 10 \%$. The well approved methods, namely HRTEM, X-ray powder diffractions and molecular weight determination give well corresponding values for the diameter, namely $18 \pm 1 \mathrm{~nm}$. Colloid growth can be directed by the concentration of gold chloride in the aqueous solution. Using the eight-fold amount of gold compared with the above mentioned synthesis, the diameter of the phosphane-stabilized colloids indeed is twice the first one.

Experiments to isolate copper, platinum, palladium, and colloids of other transition metal by this way look promising.

\section{REFERENCES}

1. G. Schmid, Chem. Unserer Zeit, 22, 85 (1988)

2. B. K. Teo, M. Hong, H. Zhang, D. B. Huang, and X. Shi, J. Chem. Soc., Chem. Comm., 1988,204

3. G. Schmid, R. Boese, R. Pfeil, F. Bandermann, S. Meyer, G. H. M. Calis, and J. W. A. van der Velden, Chem. Ber., 114, 3634 (1981)

4. G. Schmid, U. Giebel, W. Huster, and A. Schwenk, Inorg. Chim. Acta, 85, 97 (1984)

5. G. Schmid and W. Huster, Z. Naturforsch. 41b, 1028 (1986)

6. G. Schmid, B. Morun, and J. 0. Malm, Angew. Chem. i. press

7. G. Schmid, Polyhedron, 7, 2321 (1988)

8. M. N. Vargaftik, V. P. Zagorodnikov, I. P. Stolyarov, I. I. Moiseev, V. A. Likholobov, D. I. Kochubey, A. L. Chuvilin, V. I. Zaikowsky, K. I. Zamaraev, and I. Timofeeva, J. Chem. Soc., Chem. Comm., 1985, 937

9. L. J. de Jongh, H. B. Brom, G. Longoni, P. R. Nuysteren, B. J. Pronk, G. Schmid, M. P. J. van Staveren, and R. C. Thiel, Physics and Chemistry of Small Clusters, Series B: Physics 158, 807 (1987)

10. J. Turkevich, P. C. Stevenson, and J. Hillier, Discuss. Faraday Soc., 11, 55 (1951) 